\title{
L'action pédagogique à l'épreuve de l'individualisation du processus de production du savoir par les médias sociaux dans les universités au Bénin
}

\author{
Dr. Arnauld Gbaguidi, Maître de Conférences \\ Département de Sociologie-Anthropologie, \\ Université d'Abomey-Calavi, République du Bénin \\ Dr. Rodrigue Sèdjrofidé Montcho, Maître-Assistant \\ Département de Sociologie-Anthropologie, \\ Université de Parakou, République du Bénin
}

Doi:10.19044/esj.2020.v16n10p181 URL:http://dx.doi.org/10.19044/esj.2020.v16n10p181

Résumé

Les médias sociaux sont aujourd'hui au cœur du processus de production du savoir dans les systèmes éducatifs et mettent ainsi à l'épreuve l'action pédagogique. Dans ce contexte, plusieurs stratégies sont mobilisées par les acteurs en vue de faire face aux nouveaux défis. La présente recherche vise à analyser l'influence de l'action pédagogique sur le processus de production du savoir par les médias sociaux dans les universités au Bénin. Dans le cadre de cette recherche qualitative, des données sont collectées auprès de 50 personnes ressources par le biais de l'entretien et l'observation à travers les techniques d'échantillonnage à choix raisonné et par boule de neige. Il apparait que les médias sociaux contribuent à l'individualisation du processus de production du savoir. De même, l'hyper-accessibilité des médias sociaux engendre une certaine démocratisation du savoir qui remet en cause l'action pédagogique traditionnelle. Il urge donc de relever le défi d'une pédagogie numérique.

Mots clés : Action pédagogique, savoir, médias sociaux, individualisation, universités du Bénin 


\title{
Educational Action Tested by the Individualization of the Knowledge Production Process by Social Media in Universities in Benin
}

\author{
Dr. Arnauld Gbaguidi, Maître de Conférences \\ Département de Sociologie-Anthropologie, \\ Université d'Abomey-Calavi, République du Bénin \\ Dr. Rodrigue Sèdjrofidé Montcho, Maître-Assistant \\ Département de Sociologie-Anthropologie, \\ Université de Parakou, République du Bénin
}

\begin{abstract}
Social media is today at the heart of the knowledge production process in education systems and is therefore testing educational action. In this context, several strategies are mobilized by the actors in order to face the new challenges. This research aims to analyze the influence of educational action on the knowledge production process by social media in universities in Benin. As part of this qualitative research, data is collected from 50 resource persons through interview and observation through reasoned choice and snowball sampling techniques. It appears that social media contributes to the individualization of the knowledge production process. Likewise, the hyperaccessibility of social media generates a certain democratization of knowledge and which calls into question traditional pedagogical action. It is therefore urgent to raise the level of a digital pedagogy.
\end{abstract}

Keywords: Educational action, knowledge, social media, individualization, universities of Benin

\section{Introduction}

Toute éducation vise la transmission de savoirs multiples. Mais le processus de transmission du savoir dépend des objectifs, des acteurs, des méthodes et des finalités. Pour Emile Durkheim, l'éducation est « une socialisation méthodique de la jeune génération « ( Dollo C., 2017, 123). Il y a comme une dimension verticale dans le processus de transmission alors que C. $R$. Boudon (1992) parle de rationalités axiologiques, traditionnelle et cognitive. Car, selon lui, l'apprenant pèse et repèse les éléments économiques en présence et le cumul de ses choix rend davantage compte de sa trajectoire que de la seule adaptation ou mésadaptation à la culture 
scolaire. Comme pour mettre en relief les deux approches suscitées, A. Petitat fait une nuance. Selon lui,

«deux représentations coexistent en Sociologie et dans le sens commun : à l'un des pôles se tient l'individu prométhéen, qui asservit la nature, qui entre en compétition, qui poursuit librement ses intérêts sur le marché des biens ou de la formation; à l'opposé, le groupe intégré reçoit toutes les attentions, la conscience collective transcende les consciences individuelles et l'éducation consiste précisément en l'intériorisation de règles, valeurs et normes qui définissent un individu moral » Petitat A. ( 2006 ; p. 615-616).

C'est dire autrement que le processus éducatif varie ainsi entre liberté individuelle et intégration sociale. C'est du moins le constat que l'on peut faire avec l'avènement des médias sociaux qui, dans le processus de production du savoir, à travers leur hyper-accessibilité et leur dimension itérative et ubiquiste, mobilisent bien des paramètres de facilitation que remettent en cause le système traditionnel pédagogique. Ils ont permis l'avènement d'un capitalisme cognitif, « troisième espèce » du capitalisme, après le capitalisme marchand et le capitalisme industriel (Moulier-Boutang, 2007). Leurs possibilités illimitées d'interactivité et d'interconnexion donnent à la fois le vertige et un sentiment de puissance (Wertheim 1999 ; Zaleski 1997). Lorsqu'on parle de médias sociaux, il s'agit du concept englobant qui intègre aussi celui de réseaux sociaux. Car, un réseau social est associé aux plateformes en ligne qui sont davantage des supports technologiques au développement ou à l'entretien de sociabilité (G. Simmel, [1981] 1991). Les médias sociaux favorisent la communication médiatique et la conversation avec un public large. Ils sont aujourd'hui au cœur du processus de production du savoir dans les systèmes éducatifs au Bénin et mettent ainsi à l'épreuve l'action pédagogique dans sa globalité. Un numéro récent des Cahiers pédagogiques ouvrait son dossier thématique sur le numérique en disant que : «c'est la pensée complexe, chère à Edgar Morin, qui rentre dans la classe. Une forme d'enseignement qui considère le monde dans sa globalité, qui met l'élève en autonomie et en interaction pour établir des relations entre les connaissances, entre l'école et le monde, qui le responsabilise face à ses apprentissages » (Jouneau-Sion \& Touzé, 2012).

Dans ce contexte, comment l'action pédagogique se construit-elle face à l'individualisation du processus de production du savoir par les médias sociaux dans les universités au Bénin ? La présente recherche vise à analyser l'influence des médias sociaux sur le processus de production du savoir à travers l'action pédagogique dans les universités au Bénin. 


\section{Démarhe méthodologique}

Dans le cadre de cette recherche qualitative, des données sont collectées auprès de 50 personnes ressources par le biais de l'entretien et l'observation à travers les techniques d'échantillonnage à choix raisonné et par boule de neige. L'environnement du numérique et ses usages sont très dynamiques. C'est pourquoi, le modèle théorique de la désorganisation sociale combinée à celle de l'analyse stratégique de Crozier et de Friedberg a été privilégié. En effet, la théorie de désorganisation sociale met l'accent sur les facteurs fragilisant le contrôle social formel et informel et est représentée ici par W. Thomas (1920). La désorganisation sociale apparait comme la conséquence des changements rapides de l'environnement économique et social. Mieux, elle renvoie à l'idée du déclin de l'influence des valeurs collectives sur l'individu qui, se détourne de son groupe primaire. Quant à Crozier et Friedberg, le comportement des acteurs peut s'expliquer par le fait que l'acteur n'est jamais totalement contraint dans une organisation et qu'il a toujours une marge de manœuvre (M. Crozier et E. Friedberg, 1977).

Dans cet environnement, de fortes mutations engendrées par les médias sociaux, les acteurs sociaux mettent en œuvre des stratégies individuelles et collectives dans le cadre de la production du savoir. Ces stratégies ont engendré bien des déviances qui ont désorganisé le système de production de savoir à travers les mécanismes traditionnels de production du savoir dans les universités au Bénin.

\section{Résultats}

\section{Les médias sociaux comme source du savoir}

Pour produire le savoir, la démarche pédagogique vise à préparer, à conduire et à évaluer le savoir qui peut être intellectuel, pratique et comportemental. On parle généralement de savoir, de savoir-faire et de savoirêtre. Ces savoirs peuvent être catégorisés en deux groupes: les savoirs structurés qui servent à la production formelle du savoir par l'action pédagogique et les savoirs non structurés qui servent à la production non formelle et informelle du savoir dans le cadre de l'action pédagogique ou non. Plusieurs médias sociaux sont mobilisés dans le processus de production du savoir dans les universités au Bénin et sont surtout en rapport avec ceux mobilisés dans les zones francophones. Il s'agit notamment des plateformes médiatiques de masse ( Facebook, Twitter et Google+), des plateformes de contenus vidéos (YouTube et Dailymotion), les sites de réseautage professionnel ( LinkedIn, Viadeo), les blogs, les forums, le Wikipédia. Il y a aussi les précurseurs d'avant ( MySpace et Copains d'avant ), les sociaux hybrides ou spécialisés ( Foursquare, Instagram, Flick'r, Last.fm, Delicious, Pinterest), les médias sociaux les plus américains (Reddit et Digg). En dehors de ces médias sociaux, bien des moteurs de recherche sont suffisamment 
sollicités (globaux et spécifiques) et les autres plate-formes comme face book, whatsap, etc. Les médias sociaux influencent fortement tout le processus comme le décrit le tableau comparatif I.

Tableau I: Comparaison des systèmes pédagogiques de production du savoir

\begin{tabular}{|c|c|c|}
\hline $\begin{array}{l}\text { Systèmes } \\
\text { Caractéristiques }\end{array}$ & Traditionnel & Influencé par les médias sociaux \\
\hline Ressemblances & \multicolumn{2}{|c|}{$\begin{array}{l}\text { Savoirs globaux, système de plus en plus orienté vers les } \\
\text { compétences, contrats didactiques, préparation et mise en œuvre du } \\
\text { processus, évaluation sommative et formative du savoir produit }\end{array}$} \\
\hline \multirow[t]{10}{*}{ Dissemblances } & $\begin{array}{l}\text { Non utilisation ou faible } \\
\text { utilisation des outils numériques }\end{array}$ & $\begin{array}{l}\text { Forte utilisation des outils } \\
\text { numériques }\end{array}$ \\
\hline & $\begin{array}{l}\text { Faible accessibilité de } \\
\text { l'information par des guides. }\end{array}$ & $\begin{array}{l}\text { hyper-accessibilité du savoir le } \\
\text { biais des médias sociaux }\end{array}$ \\
\hline & $\begin{array}{l}\text { Savoirs diffusés par le formateur } \\
\text { ou l'enseignant }\end{array}$ & $\begin{array}{l}\text { Savoirs diffusés par les médias } \\
\text { sociaux et diversité des sources }\end{array}$ \\
\hline & Réactivité de l'apprenant & Proactivité de l'apprenant \\
\hline & $\begin{array}{l}\text { Savoir faiblement produit et } \\
\text { possibilité de déviance }\end{array}$ & $\begin{array}{l}\text { Abondance du savoir en } \\
\text { production ou produit et } \\
\text { possibilité de déviance }\end{array}$ \\
\hline & $\begin{array}{l}\text { Possibilité de capitalisation en } \\
\text { fonction des capacités du } \\
\text { formateur }\end{array}$ & $\begin{array}{l}\text { Possibilité de capitalisation en } \\
\text { fonction des capacités de } \\
\text { l'apprenant }\end{array}$ \\
\hline & Proximité physique accentuée & $\begin{array}{l}\text { Proximité physique réduite ou } \\
\text { inexistante }\end{array}$ \\
\hline & Discussion réduite & $\begin{array}{l}\text { Instantanéité dans les } \\
\text { discussions }\end{array}$ \\
\hline & Moins de visibilité & Visibilité accentuée \\
\hline & $\begin{array}{l}\text { Rapports sociaux à tendance } \\
\text { hiérarchique }\end{array}$ & $\begin{array}{l}\text { Rapports sociaux à tendance } \\
\text { horizontale }\end{array}$ \\
\hline
\end{tabular}

Source : Rodrigue S. Montcho, 2019

En dehors des facteurs de ressemblance qui sont liés au processus traditionnel de production de savoir, les médias sociaux ont apporté plusieurs éléments évolutifs. Du tableau I, il apparaît qu'il existe une mutation du processus traditionnel de production du savoir et une forte individualisation du processus de production des savoirs à travers les médias sociaux.

\section{Des mutations à la démocratisation du processus de production du savoir par les médias sociaux}

On peut oser dire que les médias sociaux ont un pouvoir qui est à la base des nombreuses mutations déduites/induites sur le processus de production du savoir dans les universités au Bénin. La figure $\mathrm{n}^{\circ} 1$ schématise cette réalité. 
Figure $\mathbf{n}^{\circ} 1$ : Mutations du processus de production du savoir chez l'apprenant dû aux médias sociaux

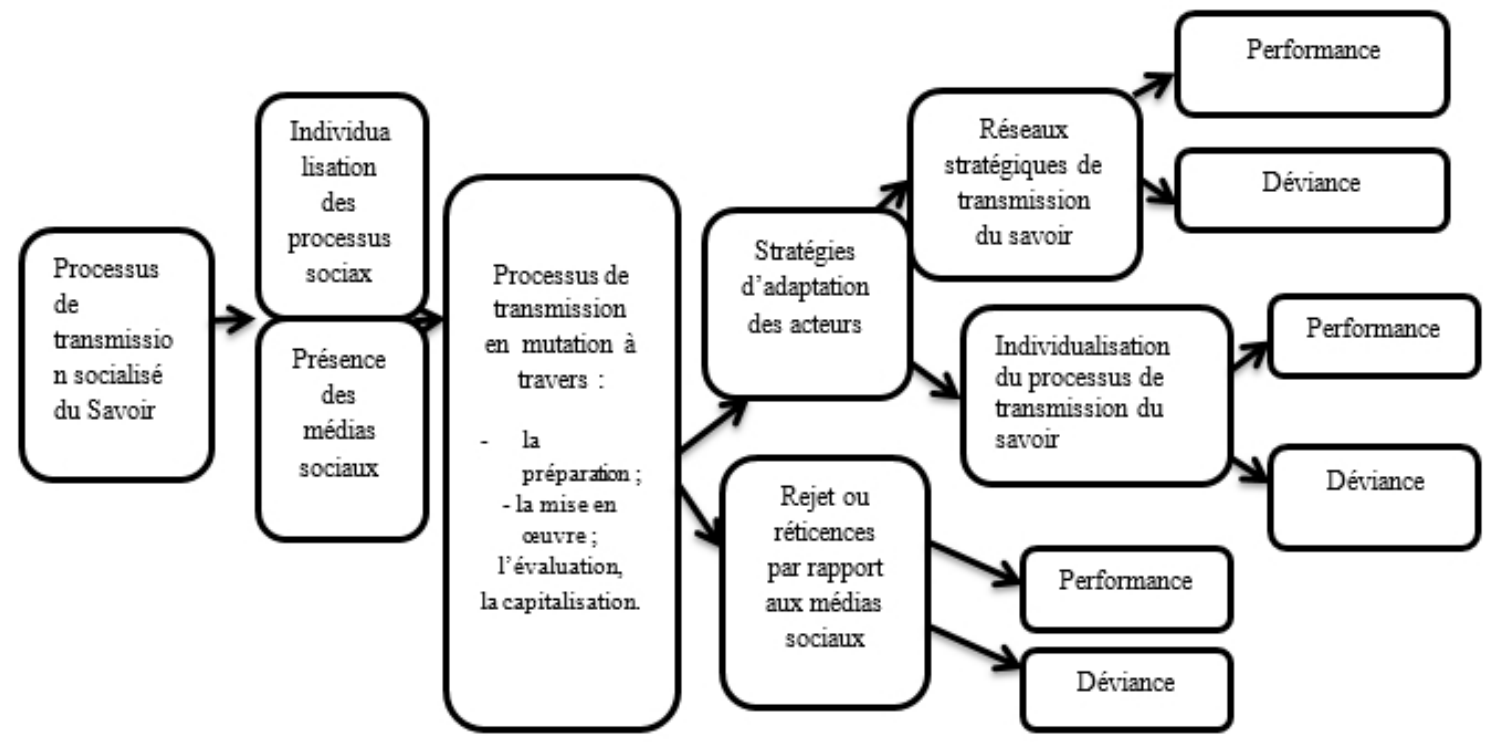

Source : Inspiré de R.S. Montcho, 2019

Comme on peut le constater à travers la figure $\mathrm{n}^{\circ} 1$, l'individualisation des processus sociaux a aussi fortement influencé l'action pédagogique. Même si la même figure permet de se rendre compte que les médias sociaux à travers leur hyper-accessibilité ont créé des mutations au niveau du processus de production du savoir chez l'apprenant. En effet, en dehors de quelques apprenants qui rejettent les médias parce que ne disposant pas des ressources, la plupart de ceux qui l'ont aujourd'hui ont accès rapidement au savoir même parfois avant le formateur ou l'enseignant comme le souligne cet apprenant : " les enseignants qui continuent de lire des cours ou d'aller chercher des syllabus, sans les contextualiser, on les supplante au cours. Parfois, nous avons le fichier que nous faisons voir à nos camarades. Les médias sociaux et les moteurs de recherche nous offrent tellement de possibilités et de facilités que nous demandons si nous avons encore besoin d'enseignants» Etudiant l'université de Parakou.

Comme le montrent les propos de cet apprenant, les meilleurs apprenants sont ceux qui internalisent utilement les savoirs sur les médias sociaux même s'il faut reconnaitre que les stratégies développées varient entre appropriations et rejets des médias sociaux dans le processus des médias sociaux. Ainsi, plusieurs groupes d'apprentissages sont-ils créés par les apprenants pour renforcer leurs compétences. Il en de même des formateurs qui créent des plates-formes pour échanger et partager leurs expériences. Ces différents groupes produisent des résultats mais se transforment en des réseaux sociaux sur des sujets sans liens utiles avec les objectifs pédagogiques de 
départ visés. De même au plan individuel, des initiatives individuelles de renforcement de capacités par les médias sociaux sont source de performance. $\mathrm{Ce}$ que l'on peut retenir, c'est que les stratégies ont leur dimension ambivalente (performance et déviance).

La performance que les médias sociaux ont sur le processus de production du savoir est liée au fait de la disponibilité et de l'agilité de l'information quoique flexible. Tout compte fait, ils renforcent les capacités. Aujourd'hui, avec les médiaux sociaux, il y a une remise en cause de la définition durkheimienne de l'éducation. Ce n'est plus forcément l'apprenant qui reçoit du formateur. L'autorité change ainsi de direction. Parler ici d'autorité dans une telle recherche pourrait surprendre surtout qu'on n'est pas dans les Sciences Politiques. Mais il s'agit ici bel et bien d'autorité. En effet, la notion telle que définie par Boudon et al (2011) est appréhendée comme une personne, une institution ou un message pour signifier qu'on leur fait confiance, qu'on accueille leur avis, suggestion ou injonction, avec respect, faveur ou du moins sans hostilité, ni résistance et qu'on est disposé à y déférer. Harendt en distinguant le concept d'autorité et celui de pouvoir affirme clairement que l'autorité est dans les mains de celui qui détient le savoir. Avec les médias sociaux, le savoir est accessible à celui consulte la bonne source. Comme le souligne Corroy, s'éduquer aux réseaux socio numériques reviendrait à cultiver ce que Corroy $(2013,5)$ a appelé l'empowerment. C'est une sorte démocratisation du savoir et qui engendre une horizontalisation du pouvoir. L'ordre pédagogique devient ainsi précaire surtout dans les structures académiques où certains enseignants continuent d'être réticents par rapport au recours utile aux médias sociaux. Car, il faut oser le dire, il y des vieux analphabètes aujourd'hui et des jeunes analphabètes surtout lorsqu'il s'agit des réseaux sociaux. Les nouveaux analphabètes sont ceux qui ne sont pas en contact avec les médias sociaux, or il y a bien des enseignants qui sont dans cette situation.

\section{Le défi d'une pédagogie adaptée aux médias sociaux}

Si le savoir est aujourd'hui disponible à travers les médias sociaux, ce qui peut faire objet de réflexion, c'est la qualité des producteurs du savoir et du savoir lui-même qui sont en cause. En effet, un savoir dans la démarche pédagogique est sensible. Comme le souligne Gaspar et al, l'appréciation de la performance fait appel à la synthèse de trois éléments principaux. Il s'agit des activités développées, les résultats obtenus et la manière dont l'action est conduite (Gaspar et al, 1993). L'action pédagogique dans le système LMD vise à rendre l'apprenant autonome, lui donner toutes les chances de réussite et l'accompagner. Le formateur ou l'enseignant dans ce processus est non seulement producteur du savoir mais aussi facilitateur. Préparer, mettre en œuvre, évaluer et capitaliser les expériences dans le processus du savoir, est 
une entreprise difficile dans le contexte où l'hyper-accessibilité des médias et en fait un pouvoir dans l'action pédagogique. Avec les medias sociaux, celui qui émet peut être à la fois récepteur et vice versa. Les apprenants peuvent ainsi produire et recevoir l'information comme l'enseignant. Le défi pédagogique dans ce contexte est complexe. Les enseignants doivent aller audelà en internalisant les outils numériques. Les enseignants ne peuvent plus produire le savoir de la même manière qu'ils avaient l'habitude de le faire. Les mutations pédagogiques liées aux médias sociaux relatives à la démarche, aux méthodes et aux matériaux cognitifs sont profondes. La qualité du savoir dépend désormais de la manière dont on internalise tous ces paramètres en vue de la performance pédagogique. Certaines initiatives prises dans les universités actuellement ne satisfont pas encore aux nouvelles exigences (bibliothèque numérique, formation à l'e learning, formation en informatique).

Dans ce cadre, les outils numériques et les médias sociaux qui sont mobilisés servent en grande partie à faire les exposés dans nos universités et n'accompagnent pas les innovations éducatives. Le défi est alors plus profond. Les médias sociaux vont à une vitesse de croisière. A l'international, il y a des innovations pédagogiques numériques qui font leur preuve. De la pédagogie 1.0, on est à la pédagogie 3.0. La Pédagogie 1.0 est une approche directive et explicite avec différentes démonstrations. La Pédagogie 2.0 est une approche collaborative par la découverte, le socioconstructivisme, le tout agrémenté par divers outils technologiques.

Le constat de l'enseignant est simple : avec une approche directive, les élèves ne retiennent pas assez et, à l'opposé, si l'approche est davantage collaborative, les élèves éprouvent des difficultés lors des évaluations où ils doivent transférer les habiletés collaboratives en contexte individuel. C'est pourquoi, selon, Girard Marc-André, la pédagogie 3.0 implique un heureux mélange des deux en permettant les interactions directes entre les élèves et en créant une atmosphère sainement compétitive. Complémentaire aux deux premières, elle permet un certain discernement professionnel et une différenciation puisque l'enseignant peut juger que, parfois, l'approche directe est plus appropriée dans une certaine proportion alors qu'à un autre moment ou dans un autre groupe, il fera davantage appel à l'approche collaborative (Girard Marc-André, 2015).

Pour qu'il en soit ainsi trois préalables sont nécessaires dans le contexte des universités du Bénin : la disponibilité des ressources numériques, le renforcement des capacités des acteurs (apprenants, formateurs, administration) et le management de l'innovation pédagogique. Ces préalables sont importants afin que les outils soient non seulement acquis, mais qu'ils ne soient pas gadgétisés du fait de leur faible internalisation lorsqu'il s'agit des fins pédagogiques. Parmi les quatre étapes d'intégration des TIC par les enseignants, on a la découverte (utilisation personnelle des outils), l'adoption 
(utilisation professionnelle mais la pédagogie reste inchangée), l'appropriation (pédagogie plus interactive) et la création (pédagogie innovante, élève acteur, producteur et créateur) selon ( Fourgous, 2012). Il précise que la majorité des enseignants se situent dans les deux premières étapes, ce qui signifie que les TIC n'ont pas d'influence notable sur la pédagogie. Pourtant, à l'heure du numérique, les compétences transversales (autonomie, adaptabilité, collaboration, créativité, etc.) sont essentielles. De même, un rapport du Becta (Condie \& Munro, 2007) révélait que bien que les enseignants aient les compétences minimales requises et bien qu'ils utilisent ces outils pour préparer leurs cours, ils sont peu enclins à y recourir dans leur salle de classe dans un contexte pédagogique. L'usage reste essentiellement bureautique et le «blended learning (apprentissage mêlant travail en présentiel et à distance) n'est pas développé (Paryono et Quito, 2010). Or, les facteurs qui encouragent l'usage des TIC (d'après les déclarations des enseignants français interrogés) sont l'accès à une diversité de ressources documentaires, la disponibilité d'un équipement adapté et la volonté d'améliorer la réussite des apprenants (Alluin, 2010). C'est pourquoi, Fourgous préconise à cet égard d'hybrider la formation initiale en s'appuyant sur les outils numériques, notamment les TBI, les ENT, les tablettes, la baladodiffusion et la visio-conférence (Fourgous, 2012). Tous ces positionnements viennent renforcer la dimension managériale de l'innovation pédagogique numérique. C'est ce management qui va mobiliser les étudiants à cette fin pour que les médias sociaux n'individualisent pas uniquement le processus de production du savoir, mais qu'ils contribuent à la performance du système éducatif et limitent les déviances observées jusque-là dans les universités au Bénin. La piste de la pédagogie 3.0 peut donc être explorée et renforcée dans le contexte béninois.

\section{Discussion}

Les médias sociaux contribuent à l'individualisation du processus de production du savoir. Le savoir n'est pas plus forcément un produit vertical venant d'un formateur et qui à travers le processus de production structuré du savoir fixant ses objectifs pédagogiques et met en œuvre un dispositif pour les atteindre. L'apprenant n'est plus forcément neutre, il va vers son autonomisation. Lucile Mera (2015) en s'appuyant sur la théorie interactionniste d'Erving Goffman (1973) qui ne distingue pas le sujet de la structure à laquelle il est intimement relié par l'interaction, montre qu'à travers les médias sociaux, les agents s' « individualise[nt] et se socialise[nt] » à la fois (C. Bonicco, 2007). Effectivement, dans la présente recherche la mise en branle des réseaux stratégiques (entre camarades ou entre collègues comme c'est le cas des réseaux intragroupes et intergroupes) dans le processus de 
production du savoir illustre le fait qu'ils n'ont pas dissout complètement des systèmes de socialisation, mais qu'il y a une resocialisation.

L’hyper-accessibilité des médias sociaux engendre ainsi une certaine démocratisation du savoir et remet ainsi en cause l'action pédagogique traditionnelle. Danah Boyd (2008) attribuent aux médias sociaux, une capacité d'affecter la manière dont les gens interagissent ensemble et un fort potentiel pour changer l'organisation de la société. Ce potentiel de rupture est également mis en avant en raison de l'impact qu'ils produiraient sur de nombreuses pratiques de la société. Ils remettent en cause l'ordre vertical dans la relation enseignant-Etudiant, formateur/apprenant et favorisent les relations horizontales plus que les relations verticales. Or, pour MouissetLacan (2012), « la symbolique de la verticalité contribue à la co-construction de sens dans la mobilisation scolaire ». L'horizontalité des relations prend de plus en plus d'importance avec l'utilisation des réseaux sociaux et « semble $s$ 'accompagner de risques sur la socialisation et la mobilisation scolaire des adolescents ». S'il est vrai que les médias sociaux favorisent la liberté d'accès à l'information et une certaine démocratisation du savoir, il urge de s'interroger sur la qualité des informations accessibles au regard des déviances que l'on observe sur les réseaux sociaux. Il y a donc une ambivalence lorsqu'il s'agit des médias sociaux dans la production du savoir (performance et déviance voir figure $\mathrm{n}^{\circ} 1$ ). Il a donc un besoin d'encadrement tout en favorisant l'initiative dans la perspective de la pédagogie 3.0.

\section{Conclusion}

L'individualisation et la démocratisation du processus du savoir par les médias sociaux mettent à l'épreuve la démarche pédagogique dans les universités du Bénin. Face aux nombreux défis de la qualité de formation et l'employabilité des produits, les médias sociaux offrent d'énormes opportunités comme ils constituent également des menaces. Il importe que le management de l'innovation pédagogique s'approprie ses facteurs afin que la pédagogie ne s'isole pas davantage des défis contemporains. C'est la contribution de cette recherche lorsqu'on observe toutes les inventions relatives aux médias sociaux quoiqu'ayant leurs limites en matière de production du savoir. La présente recherche a pour limite son caractère trop qualitatif. Mais il aura le mérite de lancer le débat dans le contexte béninois où tout le monde fait l'apologie des médias sociaux sans les internaliser à bon escient dans la pédagogie. C'est la perspective de la pédagogie 3.0 qui se dégage ainsi. 


\section{References:}

1. Alluin François, 2010, Les technologies de l'information et de la communication (TIC) en classe au collège et au lycée : Éléments d'usages et enjeux. Paris : Département des études, de la prospective et des statistiques, $\mathrm{n}^{\circ} 197$.

2. Akiyo R., Afouda A.S, Vigninou T. et N'Bessa B., 2013, Utilisation de la téléphonie mobile en République du Bénin : incidences socioéconomiques et environnementales (2013), Journal de la Recherche Scientifique de l'Université de Lomé (Togo), Vol.15, N³, Août 2013.

3. Bourdieu Pierre, 1980, «Le capital social : notes provisoires », Actes de la recherche en sciences sociales, vol. 31, janv, p. 2-3.

4. Boudon, R., 1979, La Logique du social, Paris, Presses Universitaires de France

5. Boudon R., 1978, L'inégalité des chances, Paris, A. Colin.

6. Boulier D., 2016, Sociologie du numérique, Paris, Armand Collin

7. Boyd Danah \& Ellison Nicole, 2007, « social Network sites : Definitions, History and Scholarship », Journal of computer Mediated communication, 13 (1), article 11

8. Boyd Danah, 2007, « Social Network Sites : Public, Private, or What ? » Knowledge Tree 13, May. Texte traduit eCohen D., 2000, Nos temps modernes, Paris, Flammarion, 2000.n français par Tilly BayardRichard, Paris, France.

9. Caspar P. Millet J-G, 1993, Apprécier et valoriser les hommes, Paris, Editions Liaisons.

10. Chanlat, A. et R. Bédard. 1990. « La gestion, une affaire de parole », in J.F. Chanlat et al. (dir.), L'individu dans l'organisation, les dimensions oubliées, Eska, Presses de l'Université de Laval.

11. Castells, Manuell, 1997, The Information Age : Economy, Society and Culture. Vol. I : The Rise of the Network Society ; Vol. II : The Power of Identity ; Vol. III : End of Millennium, Oxford, Blackwell.

12. Convert B., 2011, «Pour une socio-économie du numérique », in Revue Française de socio-économie, Paris, La découverte, N8.

13. Crozier M. et Friedberg E., 1977, L'Acteur et le Système, Paris, Le Seuil.

14. Corroy Laurence, 2013, «Panorama historique de l'éducation aux médias et perspectives d'actions pour l'Afrique », conférence prononcée lors du colloque sur l'éducation aux médias à Abidjan (Côte d'Ivoire) le 13 mars 2013.

15. Condie Rae \& Munro Bob, 2007, The Impact of ICT in Schools : A Landscape Review. Coventry : Becta. 
16. Dagiral E., Martin O., 2017, « Liens sociaux numériques », Sociologie [En ligne], $\mathrm{N}^{\circ}$ 1, vol. 8 | 2017, mis en ligne le 19 mai 2017, consulté le 23 novembre 2017. URL : http://sociologie.revues.org/3149

17. Detry L., 2013, « Geneviève VIDAL (dir.) (2012), La sociologie des usages. Continuités et transformations », Communication [En ligne], Vol. 32/1 |2013, mis en ligne le 03 décembre 2013, consulté le 24 novembre 2017. URL : http://communication.revues.org/4604

18. Durkheim E., 1968, Education et Sociologie, Paris PUF.

19. Durkheim E., 1969, L'évolution pédagogique en France, Paris, PUF.

20. Durkheim E., 1963, L'éducation morale, Paris, PUF.

21. Durkheim E., 1950, Leçons de sociologie, Paris, PUF.

22. Durand J-P. Weil R. (sous dir), 2006, Sociologie contemporaine, Paris, Editions Vigot.

23. Dollo Christine, 2017, Lexique de Sociologie, Paris, Dalloz.

24. Friedberg E., 1972, L'analyse sociologique des organisations, Paris, Harmattan, (2ème Édition 1987).

25. Friedberg E., 1993, Le Pouvoir et la règle, Paris, Le Seuil.

26. Fourgous Jean-Michel, 2010, Réussir l'école numérique. Paris: Ministère de l'éducation nationale.

27. Girard Marc-André, 2015, «La pédagogie 3.0, ou l'art de valider l'impact direct de l'enseignement sur la réussite des élèves » in Ecole branchée, enseigner le numérique en ligne ttps://ecolebranchee.com/la-pedagogie-3-0-ou-lart-de-validerlimpact-direct-de-lenseignement-sur-la-reussite-des-eleves/ consulté le $07 / 10 / 2019$.

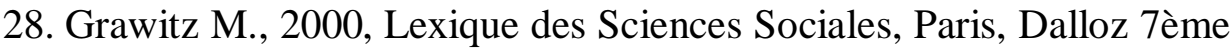
édition, $423 \mathrm{p}$.

29. Huet J-M. et al, 2010, «La téléphonie mobile facteur de développement» in L'Expansion Management Review, $\mathrm{n}^{\circ} 137$. www.cairn.info .

30. Institut National de la Statistique et de l'Analyse Economique (INSAE), 2013, Rapport du 4ème Recensement Général de la Population et de l'Habitation, Cotonou, Bénin.

31. Jouneau-Sion Caroline \& Touzé Guillaume, 2012, « Apprendre avec le numérique : Avant propos ». Les cahiers pédagogiques, ${ }^{\circ} 498$.

32. Lambert Y., 1994, « Un paysage religieux en pleine évolution», in $\mathrm{H}$. Riffaut (éd.), Les valeurs des Français, Paris, puf.

33. Mouisset-Lacan Nicole, 2012, Visibilité de la place de l'adulte (parents et enseignants) auprès de l'adolescent dans le rapport à l'apprendre: Horizontalité des pratiques d'internet et mobilisation scolaire. [thesis]. Toulouse : Université Toulouse II - Le Mirail. 
34. Neff, G., D. Stark., 2003, «Permanently Beta : Responsive Organization in the Internet Era » in P. Howard \& S. Jones, dir. Society Online : The Internet in Context. ThousandOaks : Sage Publications.

35. Paquienséguy, F., 2012, «L'usager et le consommateur à l'ère numérique » in Geneviève

36. Paryono Paryono \& Quito Benjamin Guevarra, 2010, « Meta-analysis of ICT integration in vocational and technical education in Southeast Asia ». SEAVERN Journals, vol. 2, $\mathrm{n}^{\circ} 1$.

37. Petitat André, 2006, Sociologie de l'éducation, in Sociologie contemporaine (sous dir Jean-Pi. Durand et R. Weil), Paris, éditions Vigot, p.p, 615-616.

38. Pitelis, C. N., 2013, « Towards a More 'Ethically Correct'Governance for Economic Sustainability » in Journal of Business Ethics, 1-11.

39. Philippe M., Toussaint Y, 1994, «L'intégration sociale des technologies d'information et de communication. Une sociologie des usages », Technologies de l'information et société, 6 (4).

40. Proulx S., 2015, «La sociologie des usages, et après ? », Revue française des sciences de l'information et de la communication [En ligne], 6 |, mis en ligne le 23 janvier 2015, consulté le 23 novembre 2017. URL : http://rfsic.revues.org/1230 ; DOI : 10.4000/rfsic. 1230

41. Proulx S., 1990, «La promotion sociale de la 'culture informatique' : $\mathrm{du}$ 'computer power to the people' à l'efficacité d'un nouvel outil pour le travail de bureau », Culture technique, 21, pp. 224-235.

42. Proulx S., 1988, Vivre avec l'ordinateur. Les usagers de la microinformatique. Montréal : éditions G. Vermette Inc., 1988.

43. Proulx S., «Quelle posture critique à l'ère du numérique ? », postface in Granjon, F., Lelong B., Metzger, J.L, dir., Inégalités numériques. Clivages sociaux et modes d'appropriation des TIC. Paris : éditions Hermès Science / Lavoisier, p. 251-254.

44. Puren Christian, 2009, Nouvelle perspective actionnelle et (nouvelles) technologies éducatives: Quelles convergences... et quelles divergences? Colloque Cyberlangues, Reims, 25 août 2009.

45. Redecker Christine \& Punie Yves, 2011, « Apprendre à l'heure du web 2.0 ». Administration et éducation, vol. 2011-1, n 129, p. 33-42.

46. Resta Paul \& Laferrière Thérèse, 2008, «Issues and Challenges Related to Digital Equity ». In Voogt Joke \& Knezek Gerald (dir.). International Handbook of Information Technology in Primary and Secondary Education. Vol. 20. New York : Springer US.

47. Taylor, Mark C., 2001, The Moment of Complexity : Emerging Network Culture, Chicago, University of Chicago Press.

48. Tho H. V., 2006, De la transformation de soi. L'éducation des adultes au défi des histoires de vie, Paris, Harmattan.Wertheim, Margaret, 
1999, The Pearly Gates of Cyberspace : A History of Space from Dante to the Internet, New York, W. W. Norton.

49. Zaleski, J., 1997, The Soul of Cyberspace : How New Technology Is Changing Our Spiritual Lives, San Francisco, Harpercollins. 